\title{
Complete genome sequence of Citrobacter werkmanii strain BF-6 isolated from industrial putrefaction
}

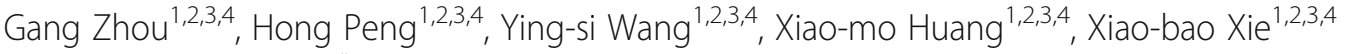 \\ and Qing-shan Shi ${ }^{1,2,3,4^{*}}$
}

\begin{abstract}
Background: In our previous study, Citrobacter werkmanii BF-6 was isolated from an industrial spoilage sample and demonstrated an excellent ability to form biofilms, which could be affected by various environmental factors. However, the genome sequence of this organism has not been reported so far.

Results: We report the complete genome sequence of $C$. werkmanii BF- 6 together with the description of the genome features and its annotation. The size of the complete chromosome is 4,929,789 bp with an average coverage of 137X. The chromosome exhibits an average $\mathrm{G}+\mathrm{C}$ content of 52.0\%, and encodes 4570 protein coding genes, 84 tRNA genes, 25 rRNA operons, 3 microsatellite sequences and 34 minisatellite sequences. A previously unknown circular plasmid designated as pCW001 was also found with a length of 212,549 bp and a G + C content of 48.2\%. 73. $5 \%, 75.6 \%$ and $92.6 \%$ of the protein coding genes could be assigned to GO Ontology, KEGG Pathway, and COG (Clusters of Orthologous Groups) categories respectively. C. werkmanii BF-6 and C. werkmanii NRBC 105721 exhibited the closest evolutionary relationships based on 165 ribosomal RNA and core-pan genome assay. Furthermore, C. werkmanii BF-6 exhibits typical bacterial biofilm formation and development. In the RT-PCR experiments, we found that a great number of biofilm related genes, such as bsmA, bssR, bssS, hms $P, \operatorname{tab} A, \operatorname{csg} A, \operatorname{csg} B, \operatorname{csg} C, \operatorname{csg} D, \operatorname{csg} E$, and csgG, were involved in C. werkmanii BF-6 biofilm formation.
\end{abstract}

Conclusions: This is the first complete genome of $\mathrm{C}$. werkmanii. Our work highlights the potential genetic mechanisms involved in biofilm formation and paves a way for further application of C. werkmanii in biofilms research.

Keywords: Citrobacter werkmanii, Complete genome, Biofilm formation, RT-PCR, Evolutionary relationships

\section{Background}

The genus Citrobacter was introduced in 1932 by Werkman \& Gillen and is a distinct group of aerobic, Gram-negative, non-spore-forming rod-shaped bacteria commonly found in water, soil, food and intestinal tracts of animals and humans $[1,2]$. Citrobacter belongs to the family Enterobacteriaceae and some strains of this genus can cause serious opportunistic infections particularly involving the urinary and respiratory tracts [3-5]. In addition, Citrobacter sp. cause enteric diseases and may also be associated with

\footnotetext{
* Correspondence: shiqingshan@hotmail.com

'Guangdong Institute of Microbiology, Guangzhou, Guangdong 510070,

People's Republic of China

${ }^{2}$ State Key Laboratory of Applied Microbiology Southern China, Guangzhou, Guangdong 510070, People's Republic of China

Full list of author information is available at the end of the article
}

extra-intestinal disorders, such as neonatal meningitis [6] and brain abscesses [7]. The species Citrobacter werkmanii was named to honor Chester $\mathrm{H}$. Werkman, an American microbiologist, who studied the fermentative production of trimethylene glycol from glycerol and proposed the genus Citrobacter [1].

Based on their physiological properties, several Citrobacter sp. were used to deal with environmental pollution or produce biological metabolites. A great number of biofilm-immobilized Citrobacter sp. have been used for bioremediation of heavy metals via the activity of an acid-type phosphatase enzyme or their ability to accumulate heavy metals [8-10]. C. werkmanii DSM17579 was considered as a new candidate for the production of 1, 3-propanediol (PDO) using cheap waste 
streams such as ligno/hemicellulosic hydrolysates [11]. Through multiple knock-outs of the dha cluster encoding PDO producing enzymes, the concentration of the toxic intermediate 3-HPA in Citrobacter species was reduced to below detection limit and the maximal theoretical PDO yield on glycerol was reached $[12,13]$.

Microbial biofilms are defined as matrix-enclosed bacterial populations that adhere to each other and to biotic or abiotic surfaces [14]. It has been found that several gene clusters, such as the curli assembly protein cluster [15-17], contribute to bacterial biofilms formation and the above process can be influenced by multiple nutritional and environmental factors $[18,19]$. In our previous study, a strain of $C$. werkmanii (named as BF-6) was successfully isolated from an industrial spoilage sample [20]. Our research indicated that the biofilms forming capacity of $C$. werkmanii BF-6 is affected by culture temperature, media, time, $\mathrm{pH}$, and the osmotic agent glucose or sucrose. Confocal Laser Scanning Microscopy (CLSM) results illustrated that biofilms structure and extracellular polysaccharide of C. werkmanii BF-6 was influenced by $\mathrm{NaCl}$ or $\mathrm{KCl}$ in a concentration-dependent manner [21]. Additionally, we also found that denser biofilms were formed by $C$. werkmanii BF- 6 in the presence of $400 \mathrm{mM} \mathrm{Ca}^{2+}$ when compared to $12.5 \mathrm{mM} \mathrm{Ca}^{2+}$ [22]. A total of 151 proteins from planktonic cells and biofilms were successfully identified after exposure of BF-6 cells to 12.5 and $400 \mathrm{mM} \mathrm{Ca}{ }^{2+}$ and categorized into different gene ontology (GO) and KEGG pathways [22]. However, the definite functions of the altered proteins and their respective signal transduction pathways were elusive owing to the lack of $C$. werkmanii BF-6 genome information.

Up to now, there is only one submitted draft genome of C. werkmanii NBRC 105721 in the NCBI genome databases. Furthermore, the genomic structure and basic properties of C. werkmanii NBRC 105721 have not been reported. In this study, we sequenced the complete genome of $C$. werkmanii BF-6 and compared it with that of C. werkmanii NBRC 105721 and other strains of the genus Citrobacter.

\section{Results}

\section{General genomic features}

The whole genome sequence of $C$. werkmanii BF-6 was obtained with no gaps by the Illumina Hiseq 4000 and Pacbio RSII platforms. The main features of the $C$. werkmanii BF-6 genome and the $\mathrm{pCW} 001$ plasmid are summarized in Table 1 . The complete genome sequence of BF- 6 comprises a 4,929,789 bp circular chromosome containing 4570 protein-coding genes, 3 microsatellite sequences, 34 minisatellite sequences, 25 rRNA genes and 84 tRNA genes with an average $\mathrm{G}+\mathrm{C}$ content of $52.0 \%$ (Table 1; Fig. 1a). We also discovered a plasmid (named as
Table 1 General features of the C. werkmanii BF-6 genome and the pCW001 plasmid

\begin{tabular}{lll}
\hline Items & Element and characteristics & Value \\
\hline Chromosome & Size (bp) & $4,929,789$ \\
& Proteins & 4570 \\
& Genes & 4846 \\
& G + C content (\%) & $52.0 \%$ \\
& tRNA genes & 84 \\
& rRNA operons & 25 \\
& Microsatellite sequences & 3 \\
& Minisatellite sequences & 34 \\
Plasmid pCW001 & Size (bp) & 212,549 \\
& G + C content (\%) & $48.2 \%$ \\
& Proteins & 250 \\
& Genes & 263 \\
\hline
\end{tabular}

pCW001) from BF-6 cells, which is 212,549 bp long with an average $G+C$ content of $48.2 \%$ and contains 250 proteins including RepB family plasmid replication initiator protein, locus_tag: B2G73_RS24605, homologous protein No.: WP_007372338.1; plasmid stability protein, locus_tag: B2G73_RS25160, homologous protein No.: WP_009652914.1; plasmid stabilization protein, locus tag: B2G73_RS25165, homologous protein No.: WP_00 7372199.1; etc. (Table 1; Fig. 1b).

\section{Comparative genomics of Citrobacter sp. strains}

To understand the evolutionary relationship of $C$. werkmanii BF-6 with other Citrobacter sp. strains, a $16 \mathrm{~S}$ rRNA Neighbor-Joining phylogeny of BF-6 with 11 other Citrobacter sp. was performed. Phylogenetic analysis revealed close evolutionary relationship of $C$. werkmanii BF-6 with C. werkmanii NRBC 105721 (Fig. 2a). To further confirm this observation, a maximum-likelihood tree of the C. werkmanii BF-6 and 11 reported Citrobacter sp. complete genomes was also created based on corepan genome analysis. These results also showed that $C$. werkmanii BF-6 is closely related to $C$. werkmanii NRBC 105721 (Fig. 2b). These results point towards a common evolutionary path between these two species of Citrobacter.

\section{Core and pan genes}

We compared the gene content of the $C$. werkmanii BF6 genome with other Citrobacter sp. reference genomes using BLAST 2.2.26. The number of core genes decreased with the addition of new strains, whereas the pan genes continued to expand after addition of the 11 Citrobacter sp. genomes (Fig. 3a and b). The core Citrobacter genome contains 3450 genes, and the pan genome consists of 8356 genes shared among the 12 strains, including $C$. werkmanii BF-6. Detailed information about the core genes is listed in Additional file 1: Table S1. Many of 

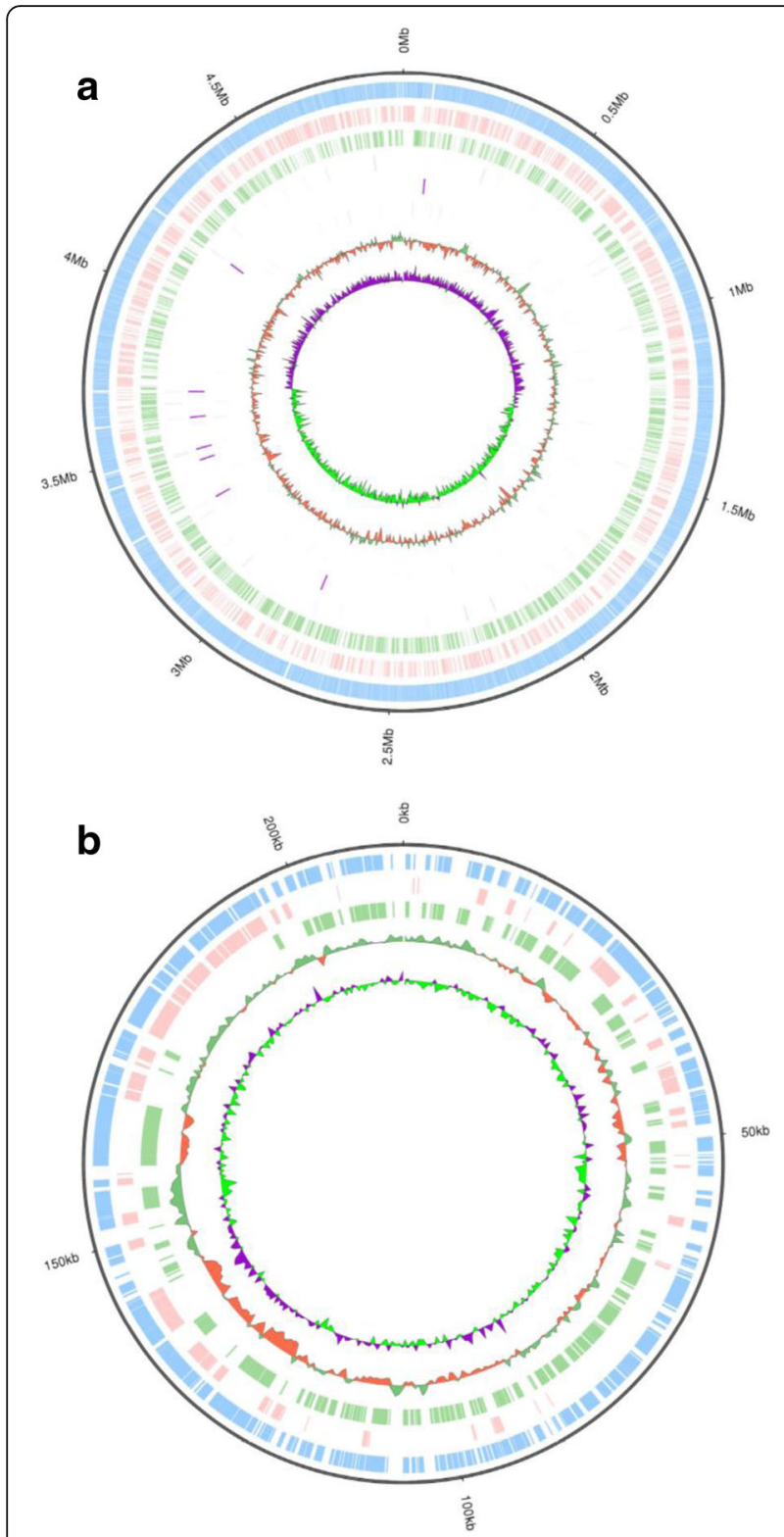

Fig. 1 Circular representation of the complete genome of C. werkmanil BF-6 (a) and the plasmid pCW001 (b) displaying their relevant features, respectively. a From the inner- to the outermost circle: circle 1, GC skew (positive GC skew in green and negative GC skew in purple); circle 2, GC content; circle 3, sRNA; circle 4, rRNA; circle 5, tRNA; circle 6, genes on reverse strand; circle 7, genes on forward strand; circle 8 , all annotated genes; circle 9, genome size. $\mathbf{b}$ The circular map of pCW001 was visualized in CGView. The features are the following from center to outside: GC skew, GC content, genes on reverse strand, genes on forward strand, all annotated genes, and plasmid size

the C. werkmanii BF-6 strain specific genes (239 and 30 genes located on the chromosome and plasmid pCW001, respectively) were hypothetical genes, the detailed information of which is provided in Additional file 2: Table S2. A heatmap after core gene deletion is also depicted (Fig. 3c). The above results demonstrated that $C$. werkmanii BF-6 and C. werkmanii NRBC 105721 shared the greatest similarity, providing molecular evidence for the similarities in phenotypes.

\section{Functional annotation of the C. werkmanii BF- 6 genome} $\mathrm{GO}$ terms were assigned to $C$. werkmanii $\mathrm{BF}-6$ genes for functional categorization. A total of 3361 genes were categorized into 42 subcategories belonging to the three main categories of biological process, cellular component and molecular function (Additional file 3: Figure S1). Among the 19 subcategories of biological process, most genes were assigned to cellular process, metabolic process and single organism process. In the cellular component category, a high percentage of genes belonged to cell, cell part, and membrane subcategories. Within the molecular function category, a majority of GO terms were grouped into catalytic activity, binding, and transporter activity subcategories. These GO annotations demonstrated that a wide variety of metabolic, structural, regulatory and transporter proteins were encoded by the $C$. werkmanii BF-6 genes.

To understand the intracellular metabolic pathways and functions of gene products, the genes were mapped to their corresponding terms in the KEGG pathway database. A total of 3453 genes were assigned to 40 KEGG pathways (Additional file 4: Figure S2). Carbohydrate metabolism $(737,21.3 \%)$ was the largest category, followed by signal transduction $(533,15.4 \%)$, overview $(439,12.7 \%)$, membrane transport $(424,12.3 \%)$, infectious disease (413, $12.0 \%)$, and amino acid metabolism (402, 11.6\%). These functional annotations of the genes of $C$. werkmanii BF-6 provide a basis for exploring specific biological processes, functions, subcellular localization, and pathways of gene products in genome research.

The COG database classified gene products into different clusters of orthologous groups. In this study, 4234 genes of C. werkmanii BF-6 were classified into 4 first classes and $23 \mathrm{~s}$ classes of functional categories (Additional file 5: Figure S3). The top three categories were: carbohydrate transport and metabolism (474, 11.2\%), amino acid transport and metabolism (433, 10.2\%), and transcription $(387,9.1 \%)$. At the same time, the smallest group was RNA processing and modification (1, 0.02\%).

\section{CLSM observation of BF-6 biofilms}

In our previous study, we found that BF- 6 possesses a high capacity for biofilm formation. Therefore the morphology, topography and architecture of BF-6 biofilms grown on glass cover slips over a period were observed using CLSM. As shown in Fig. 4, typical and denser biofilms were constructed by BF- 6 on the second and fourth days, respectively. Biofilms dispersal was observed on the sixth day. Quantitative analysis of the BF-6 biofilms was conducted using COMSTAT 2.0 with at least 5 independent 

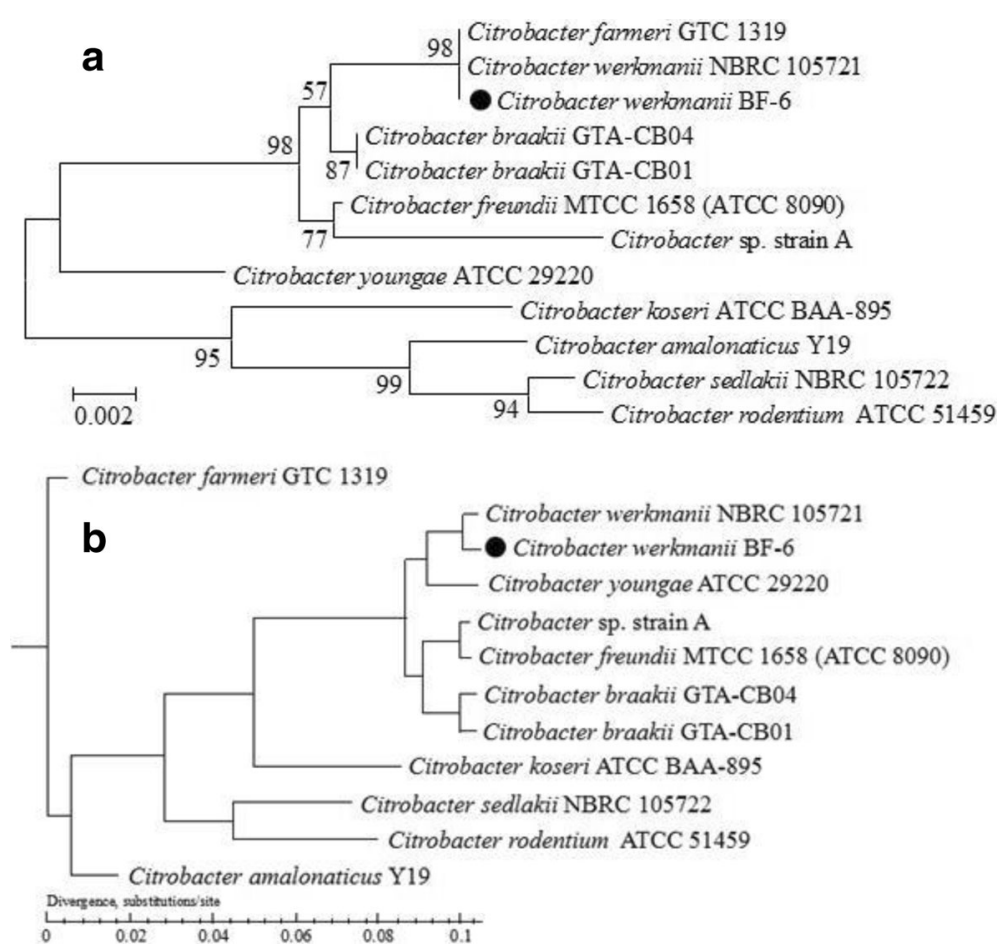

Fig. 2 Dendrogram indicating the phylogenetic relationship of C. werkmanii BF-6 with other Citrobacter sp. a 16 s ribosomal RNA phylogeny. This figure was constructed using MEGA 6.0 based on the Neighbor-Joining method. Bootstrap values (expressed as percentages of 1000 replicates) are shown at branch points; (b) Whole-genome phylogeny based on core-pan genome analysis

scans for each sample in the CLSM experiment. The highest values of total biomass, maximum and average thickness, were found on the fourth day $(p<0.05$; Table 2$)$. No differences in these values were observed on the second and sixth days $(p>0.05$; Table 2$)$. The results analyzed by COMSTAT 2.0 were thus inconsistent with the CLSM observations.

Relative gene expression of selected biofilm formation genes As reported in existing literature, several genes are involved in the process of biofilms formation [23, 24]. Therefore, partial genes related to biofilms formation in the genome of $C$. werkmanii BF- 6 were selected and their expression levels in the planktonic cells and biofilms (2 days old) were calculated using RT-PCR (Additional file 6: Table S3). The 12 selected genes and their relative location sites on the chromosome of BF- 6 are illustrated in the Fig. 5a. The relative expression levels of all tested genes except $\operatorname{csg} F$ were up-regulated, suggesting that they are involved in biofilms formation (Fig. 5b). Meanwhile, the changed expression trend of the selected biofilms formation genes on the fourth and sixth days was similar to that on the second day (data not shown).

\section{Discussion}

In this study, the complete genome of $C$. werkmanii BF-6 was sequenced with no gaps and comparative genome analyses were also conducted within Citrobacter sp. To our knowledge, this is the first report of the complete genome map of $C$. werkmanii.

The genus Citrobacter is classified into 11 genomospecies based on DNA hybridization: C. freundii, C. koseri, C. amalonaticus, C. farmeri, C. youngae, C. braakii, $C$. werkmanii, $C$. sedlakii, $C$. rodentium, $C$. gillenii, and $C$. murliniae [25-27]. In our study, we found that the size of genome $C$. werkmanii BF-6 was 4,929,789 bp which is similar to that of C. werkmanii NBRC 105721 (4,947,997 bp) and C. farmeri GTC 1319 (4,929,495 bp, Table 3). Phylogenetic analysis revealed close evolutionary relationship of BF-6 with C. farmeri GTC 1319 and C. werkmanii NRBC 105721 based on $16 \mathrm{~S}$ ribosomal RNA analyses. However, $C$. werkmanii BF-6 and $C$. werkmanii NRBC 105721 shared the closest evolutionary relationship according to a core-pan genome comparison (Fig. 1). Although C. werkmanii BF-6 and C. werkmanii NRBC 105721 have the closest evolutionary relationship, BF-6 has a plasmid of pCW001 that is not found in NRBC 105721 (Table 1; Fig. 2). Further research is needed to determine the function of the plasmid.

Meanwhile, general function analysis of the BF-6 genome demonstrated that the genes identified were primarily involved in carbohydrate transport and metabolism (Additional file 3: Figure S1, Additional file 4: Figure S2 and Additional file 5: Figure S3). Our previous study 

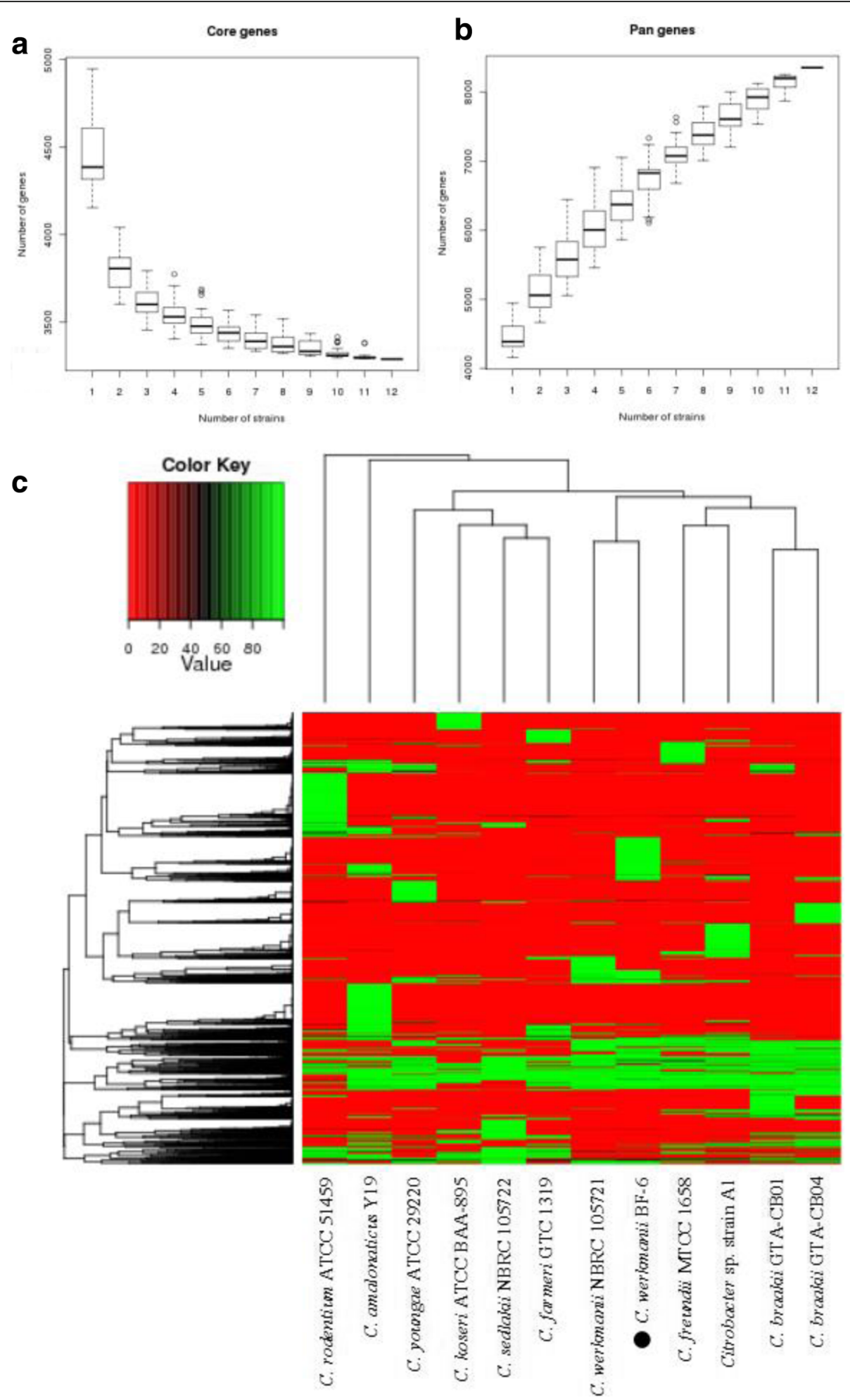

Fig. 3 Analysis of the core and pan genome of Citrobacter sp. Dilution curve of bacterial core (a) and pan (b) genes. Heatmap was depicted after core gene deletion with the coverage cutoff $>0.5$ (c) 


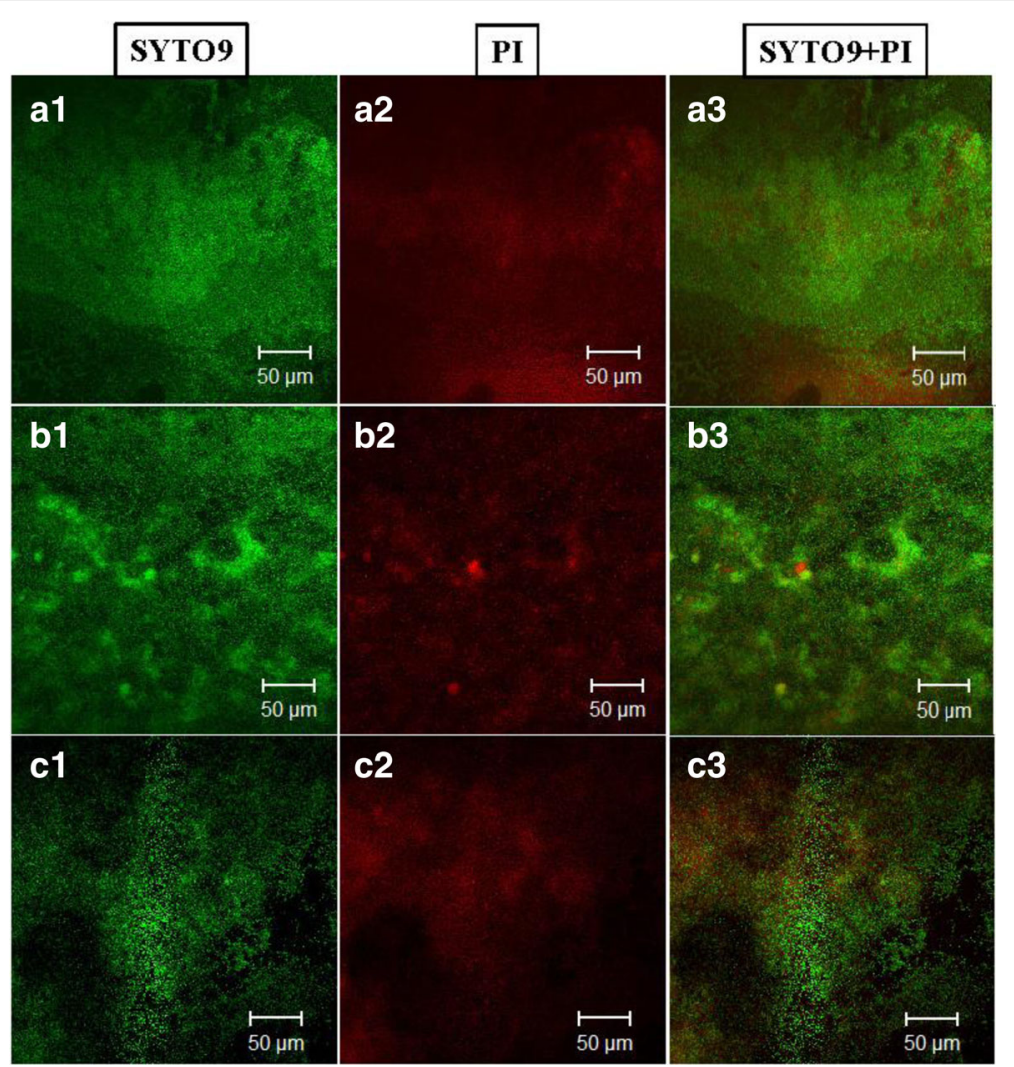

Fig. 4 CLSM images of C. werkmanii BF-6 biofilms grown on glass slides and stained with SYTO9 and PI. A1, A2, A3: 2 days; B1, B2, B3: 4 days; C1, C2, C3: 6 days. Scale bar $=50 \mu \mathrm{m}$

showed that glucose, mannitol, sorbitol, arabinose, but not inositol, sucrose and melibiose, can be used as carbon sources for growth [20]. In addition, we also found that both planktonic growth and biofilm formation of C. werkmanii BF-6 reduced with increasing concentration of glucose. At higher concentrations of glucose $(800$ and $1600 \mathrm{mM}$ ), most of the planktonic and biofilm growth was repressed [21]. These results demonstrated that glucose can be used by $C$. werkmanii BF- 6 as a carbon source to grow or form biofilms only at lower concentrations, whereas higher concentrations may cause osmotic stress and inhibit growth. In Escherichia coli, the repressive effect of glucose is exerted through catabolite repression via the cAMP-CRP system [28]. Enzyme II A (EIIA)

Table 2 Quantitation of biofilm architecture

\begin{tabular}{llll}
\hline Items & 2 days & 4 days & 6 days \\
\hline Total biomass $\left(\mu \mathrm{m}^{3} / \mu \mathrm{m}^{2}\right)$ & $19.86 \pm 3.11^{\mathrm{b}}$ & $24.84 \pm 1.60^{\mathrm{a}}$ & $19.67 \pm 2.14^{\mathrm{b}}$ \\
Maximum thickness $(\mu \mathrm{m})$ & $9.20 \pm 1.10^{\mathrm{b}}$ & $13.20 \pm 1.10^{\mathrm{a}}$ & $8.40 \pm 0.89^{\mathrm{b}}$ \\
Average thickness $(\mu \mathrm{m})$ & $7.00 \pm 1.51^{\mathrm{ab}}$ & $8.42 \pm 1.55^{\mathrm{a}}$ & $6.20 \pm 1.38^{\mathrm{b}}$ \\
\hline
\end{tabular}

"Quantitation of biofilm parameters, including total biomass, maximum thickness, average thickness, were evaluated using COMSTAT. The results are means of datasets obtained from analysis of five CLSM images acquired at random positions in each of the biofilms. Standard deviations (SD) are also shown at the end of each of the means. Different superscript letters denote significant differences within a row (Tukey's HSD: $P<0.05$ ) plays a central role in this system and there are different catabolite-specific EIIAs in a single cell [29]. In the $C$. werkmanii BF-6 genome, a great number of specificities of EIIA enzymes to different sets of catabolites were also found, such as PTS fructose transporter subunit IIC (Accession No.: WP_042306972.1), PTS fructose transporter subunit IIBC (WP_079223421.1), PTS mannose transporter subunit IID (WP_003833769.1), PTS mannose/ fructose/sorbose transporter subunit IIC (WP_005122174.1), PTS glucose transporter subunit IIA and IIBC (WP_042 312726.1 and WP_003036277.1), PTS trehalose transporter subunit IIBC (WP_042312710.1). Hence, we propose that carbon catabolite repression (CCR), a regulatory phenomenon by which the expression of genes for the use of secondary carbon sources and the activities of the corresponding enzymes is reduced in the presence of a preferred carbon source, also exists in C. werkmanii BF-6.

Typical biofilm development involves five stages: initial attachment of cells to the surface, production of EPS resulting in more firmly adhered "irreversible" attachment, early development of biofilm architecture, maturation of biofilm architecture, and dispersion of single cells from the biofilm [30]. From the CLSM images (Fig. 4) and biofilm architecture data (Table 3), we conclude that $C$. werkmanii BF-6 has a typical biofilm development 


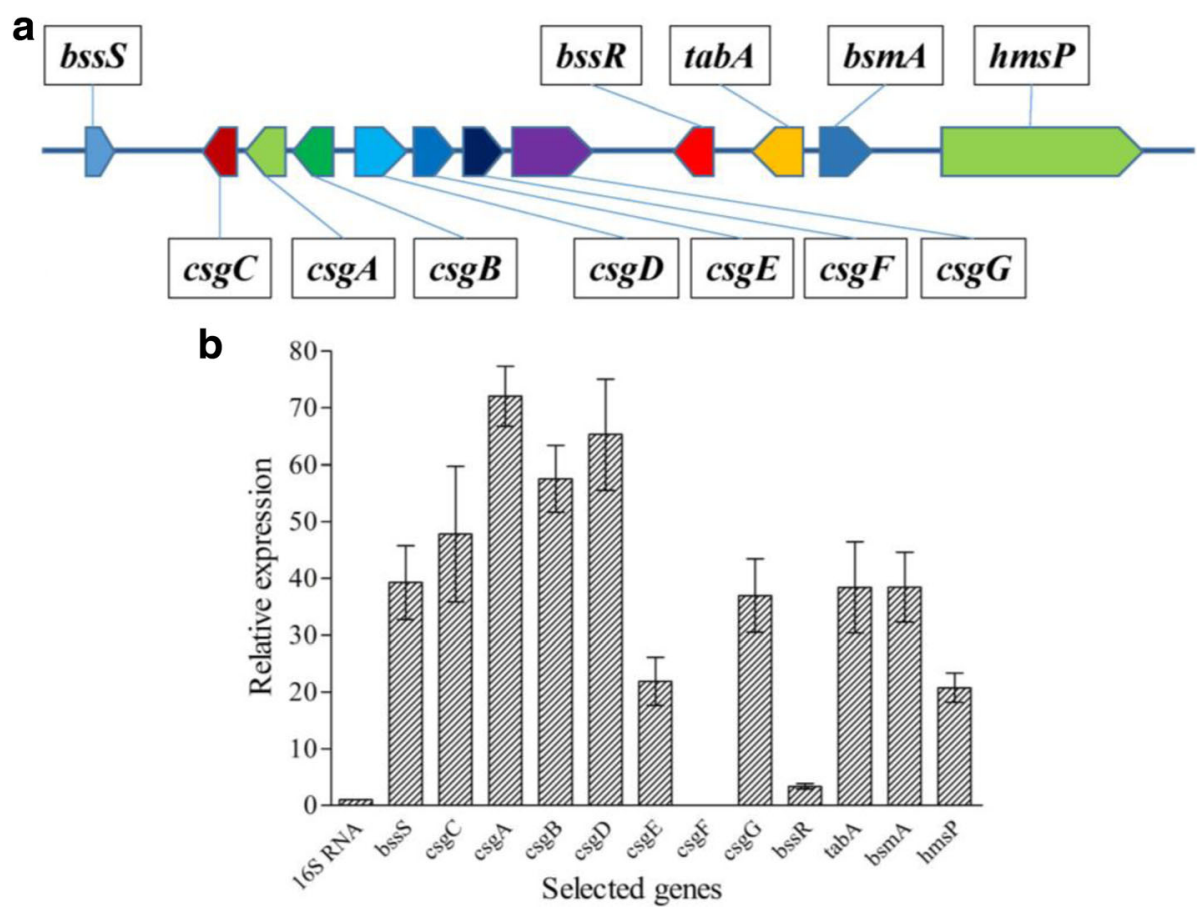

Fig. 5 Schematic representation of the curli assembly protein gene cluster and other biofilm-formation related genes on the chromosome of $C$. werkmanii BF-6 (a), and their relative expression levels in the 2-day old biofilms as determined by qRT-PCR (b). All assays were performed in triplicate; mean values and standard deviations are shown

process and structure. A large number of genes are involved in biofilms formation and development [31, 32]. It has been reported that $b s m A$, a quorum-sensingregulated gene, is engaged in fine-tuning the formation of cell aggregates at a specific point in biofilm formation and development [33,34]. Another pair of genes, $b s s R$ and $b s s S$, appear to be global regulators of the uptake and export of signaling pathways, including quorum sensing [35]. Quorum sensing controls biofilm formation through modulation of Cyclic di-GMP levels, a signaling molecule that governs the transition between planktonic and biofilm states [36, 37]. HmsP, a putative phosphodiesterase, control Hms-dependent biofilm formation: a critical residue (E506) of HmsP within the EAL domain that is required for inhibition of biofilm formation is also essential for its phosphodiesterase activity in Yersinia pestis $[38,39]$. Overexpression of YjgK (TabA), a component of the Toxin-Antitoxin system, decreased biofilm formation at $8 \mathrm{~h}$ and increased biofilm formation at $24 \mathrm{~h}$; as expected deletion of $y j g K$ also affected biofilm formation

Table 3 General features of Citrobacter sp. strains

\begin{tabular}{|c|c|c|c|c|c|c|c|}
\hline Organisms & Accession number & Size (Mb) & GC content (\%) & Number of genes & tRNA & rRNA & Reference $^{*}$ \\
\hline Citrobacter sp. strain A1 & AKTT00000000 & $5,096,012$ & $51.8 \%$ & 4836 & 63 & 4 & [57] \\
\hline Citrobacter freundii MTCC 1658 & ANAV00000000 & $5,001,265$ & $51.6 \%$ & 4691 & 70 & 10 & [58] \\
\hline Citrobacter rodentium DBS100 (ATCC 51459) & JXUN00000000 & $5,385,810$ & $54.7 \%$ & 5268 & 80 & 38 & [59] \\
\hline Citrobacter youngae ATCC 29220 & NZ_ABWL00000000.2 & $5,150,259$ & $52.7 \%$ & 4950 & 79 & 14 & - \\
\hline Citrobacter koseri ATCC BAA-895 & NC_009792 & $4,720,462$ & $53.8 \%$ & 4515 & 83 & 22 & - \\
\hline Citrobacter braakii GTA-CB01 & JRHK00000000 & $5,234,166$ & $51.9 \%$ & 4954 & 85 & 22 & [60] \\
\hline Citrobacter braakii GTA-CB04 & JRHL00000000 & $5,036,963$ & $52.0 \%$ & 4698 & 23 & 23 & [60] \\
\hline Citrobacter amalonaticus Y19 & NZ_CP011132 & $5,584,358$ & $53.4 \%$ & 5440 & 84 & 22 & - \\
\hline Citrobacter sedlakii NBRC 105722 & NZ_BBNB01000001 & $4,631,466$ & $54.7 \%$ & 4463 & 74 & 6 & - \\
\hline Citrobacter farmeri GTC 1319 & NZ_BBMX01000006 & $4,929,495$ & $53.4 \%$ & 4789 & 66 & 5 & - \\
\hline Citrobacter werkmanii NBRC 105721 & NZ_BBMW00000000 & $4,947,997$ & $52.1 \%$ & 4860 & 68 & 5 & - \\
\hline
\end{tabular}

"**": the "-" indicate that the genomes of selected Citrobacter sp. strains have not been published 
in E. coli by increasing biofilm formation after $8 \mathrm{~h}$ and decreasing biofilm formation after $24 \mathrm{~h}$ [40]. In this study, we found that the relative expression levels of $b s m A, b s s R$, $b s s S$, hmsP, and tabA were increased in the two-day old biofilms of C. werkmanii BF-6 (Fig. 5), suggesting that these genes are involved in biofilms formation. Curli assembly is guided by the products of seven curlispecific genes ( $c s g$ ) encoded on two divergently transcribed operons, csgDEFG and $\operatorname{csg} B A C$. $\operatorname{csg} D$ is the master regulator of curli biogenesis and is required for transcription of the $\operatorname{csgBAC}$ operon [41]. Meanwhile, initial steps of biofilm development require transcription of genes involved in reversible attachment and motility, while the subsequent steps require genes involved in the irreversible attachment of bacteria [42]. In addition, the second irreversible step might require the synthesis of adhesive organelles, such as the curli fibers (csg genes) [43]. In this study, all genes of csg cluster except csgF were found to be up-regulated upon RT-PCR analysis (Fig. 5). csgF associates with the outer membrane and is required for cell association of the minor curli fiber subunit $\operatorname{csg} B$. The miss detection of $\operatorname{csgF}$ may be due to the limitations of detection sensitivity of RT-PCR used in this study.

\section{Conclusions}

In this study, the first complete genome of C. werkmanii was sequenced and reported. We found that the size of the complete chromosome of $C$. werkmanii BF-6 is $4,929,789$ bp with an average $\mathrm{G}+\mathrm{C}$ content of $52.0 \%$, and encodes 4570 protein coding genes. Meanwhile, a previously unknown circular plasmid designed as pCW001 was also found with a length of 212,549 bp and a $\mathrm{G}+\mathrm{C}$ content of $48.2 \%$. Based on $16 \mathrm{~S}$ ribosomal RNA and core-pan genome assay, we found that $C$. werkmanii BF-6 and C. werkmanii NRBC 105721 exhibited the closest evolutionary relationships. Furthermore, CLSM observation showed that $C$. werkmanii BF- 6 possessed a typical biofilm developing capability and exhibited a high and stable biofilms. In the RT-PCR experiments, we also found that a great number of biofilm related genes, such as $b s m A, b s s R, b s s S, h m s P$, tabA, csg gene cluster, were involved in C. werkmanii BF-6 biofilm formation. Overall, the elucidation of the complete genome of $C$. werkmanii BF-6 provides a stable molecular foundation for genetic modification and industrial utilization of this strain.

\section{Methods}

\section{Bacterial strain and culture conditions}

C. werkmanii BF-6 was previously isolated from an industrial putrefaction sample and has been deposited at the Guangdong Culture Collection Center (Guangzhou, Guangdong, China) under the accession number GDMCC 1.1242. BF-6 was routinely inoculated into Luria Bertani (LB) medium and cultured at $30{ }^{\circ} \mathrm{C}$ and $165 \mathrm{rpm}$ for the required time in a shaker incubator. All chemicals used in this study were reagent grade and purchased from Sigma (St Louis, MO, USA) unless indicated otherwise.

\section{Whole-genome sequencing}

Cells of $C$. werkmanii BF-6 were harvested in exponential phase at an optical density of 0.6 at $600 \mathrm{~nm}$. The genomic DNA of BF-6 was extracted using QIAamp DNA Mini Kit (Qiagen, CA, USA) according to the manufacturer's protocol and fragmented randomly through Covaris or Bioruptor method. After fragmentation, the overhangs of the shortfragments were converted into blunt ends using T4 DNA polymerase (NEB, Beijing, China), Klenow DNA Polymerase (NEB) and T4 PNK (NEB). Sequencing adaptors were ligated to their $3^{\prime}$ ends and target segments that met the required length were isolated by gel electrophoresis after PCR amplification. The prepared libraries were sequenced using Illumina Hiseq 4000. To construct the complete genome of BF-6, the extracted genome was also sequenced by the Pacbio RSII platform. Briefly, library preparation (SMRTbell) was performed according to the manufacturer's protocols (Pacific Biosciences, Menlo Park, CA, USA). The prepared SMRTbell library was quantified using Qubit 2.0 Fluorometer (Invitrogen, Shanghai, China).

\section{Genome assembly and annotation}

For quality control of the sequencing data, clean reads were obtained by removing reads with low quality, mismatched reads and duplicated reads using PreAssembler Filter v1 of SMRT analysis [44]. De novo assembly was performed with the help of SOAPdenovo 2.04 [45, 46], SMRT Analysis software v2.2.0 (Pacific Biosciences) featuring HGAP 2 [44], with subsequent correction by quiver in addition to Gepard v1.30 [47]. Putative open reading frames (ORFs) were predicted using Glimmer 3.02 [48] and GeneMark.hmm [49], and putative ORF functions were analyzed by BLASTP (Coverage $\geq 40 \%$ and identity $>40 \%$ ) and InterProScan [50]. All ORFs were also translated and aligned using the NCBI nonredundant database, SwissProt database, Gene Ontology (GO), Clusters of Orthologous Groups (COG) and Kyoto Encyclopedia of Genes and Genomes (KEGG).

\section{Comparative study on the core and pan genome}

The comparative study on the core and pan genome was conducted according to the previlusly reported methods $[51,52]$. Briefly, the gene set in Citrobacter sedlakii NBRC 105722 was selected and regarded as the Reference and the gene sets in the other 11 Citrobacter sp. genomes were considered as the Query. The Query genes in each genome were aligned against the Reference genes in reference strain using BLAST v2.2.26 (http://blast.ncbi.nlm.nih.gov/Blast.cgi) and the blast results were filtered by their length and identity. Each gene 
in the Reference and Query gene sets was calculated with the BLAST Coverage Ratio (BCR) using the following formula: $\mathrm{BCR}($ Reference $)=($ Match $/$ Length $($ Reference $)) \times 100 \%$ and BCR $($ Query $)=($ Match/Length $($ Query $) \times 100 \%$. Aligned by the genes from samples, the BCR values of genes from pan gene pool were calculated for each sample, and then the coverage array was generated for the pan gene pool. If the $B C R$ value of the gene was larger than the setting value in each sample, the gene was the core gene. If the gene was predicted from assemble result, the blast results should be filtered, and the sequence should be removed if the number of $\mathrm{N}$ was large than the setting $(30 \%$ as fault setting) in the gene.

\section{Phylogenetic analysis and genomic comparison}

To understand the evolutional relationship between $C$. werkmanii BF-6 and 11 Citrobacter sp. strains, a phylogenetic tree was constructed using MEGA6 according to their $16 \mathrm{~S}$ ribosomal RNA. For comparative genomic analysis of the BF- 6 strain, genome sequences of these 11 Citrobacter sp. strains (Table 2) were downloaded from NCBI. The subprogram phyml of TreeBeST (http://tree soft.sourceforge.net/treebest.shtml) was used to construct a phylogenetic tree with the default parameters. Non-parametric bootstrap analysis with thousand resamplings was conducted to obtain bootstrap values for all branches.

\section{Confocal laser scanning microscopy for analysis of biofilms}

Based on our preliminary analysis, we found that BF-6 possessed a high capability for bacterial biofilm formation. To detect biofilm formation development, we observed the change of morphology and three-dimensional structures over a period using Confocal Laser Scanning Microscopy (CLSM) according to previously described methods [22, 53]. Briefly, a portion of coverslip was placed in a 24-well microtiter plate inoculated with aliquots of $2 \mathrm{ml} \mathrm{BF-6}$ overnight cultured supernatant with an optical density of 0.05 . The microtiter plates were then placed into a static incubator at $30{ }^{\circ} \mathrm{C}$ for 2,4 or 6 days. On the indicated day, the glass slides were gently taken out from microtiter plates and washed gently with deionized water to remove loosely attached planktonic cells. The attached biofilms on the coverslip was stained with $5 \mu \mathrm{M}$ SYTO9 fluorescent dye (Invitrogen, Carlsbad, CA, USA) and $30 \mu \mathrm{M}$ propidium iodide (Sigma Chemical Co., St. Louis, MO, USA) for at least $15 \mathrm{~min}$. Subsequently, the slides were washed gently again with deionized water and the stained biofilms were visualized using CLSM (LSM 710 Zeiss, Jena, Germany). Finally, quantification of biofilm structures was evaluated using COMSAT 2.0 software based on the obtained serial CLSM figures $[54,55]$.

\section{RT-PCR}

The planktonic cells and biofilms of $C$. werkmanii BF-6 were cultured in 24-well microtiter plates as described in the CLSM assay section above. On the indicated days, the planktonic bacteria were pipetted into a new plastic tube and the attached biofilms on the slides were also scraped into a new tube, respectively. After washing with diethylpyrocarbonate-treated water (DEPC-water) and centrifugation $(10,000 \mathrm{rpm} \times 5 \mathrm{~min})$ for three times, total RNAs of the collected samples were isolated using EasyPure RNA Kit (TransGen Biotech, Beijing, China) and converted into cDNA using the TransScript OneStep gDNA Removal and cDNA Synthesis SuperMix (TransGen) according to the manufacturer's instructions. Ten-fold dilutions of the cDNA samples were used as templates for assessing the transcriptional levels of the 12 selected biofilms formation related genes via qRTPCR with paired primers and 16S rRNA as an internal standard and sterile deionized water as the negative control (Additional file 6: Table S3). All qRT-PCRs were performed on a Mastercycler ep realplex (Eppendorf, Hamburg, Germany) with TransStart Top Green qPCR SuperMix (TransGen) following the manufacturer's guides. The transcription level of each gene in the cDNA preparation was assessed using the $2^{-\Delta \Delta \mathrm{Ct}}$ method [56]. The ratio of the transcription level of a given biofilms formation gene from BF- 6 biofilms or planktonic cells grown at the second, fourth or sixth days to that from the planktonic BF- 6 collected at the second day was defined as the relative transcription level.

\section{Additional files}

Additional file 1: Table S1. Core genes of 12 Citrobacter sp. (XLS 3788 kb) Additional file 2: Table S2. C. werkmanii BF-6 strain specific genes. (XLS $52 \mathrm{~kb}$ )

Additional file 3: Figure S1. Gene ontology (GO) analysis of $C$. werkmanii BF-6 genome. GO analysis of C. werkmanii BF- 6 genome based on GO second level terms, corresponding to 3361 genes for their predicted involvement in biological process (blue), cellular component (brown) and molecular function (yellow). Classified gene objects are depicted as gene numbers. (DOCX 44 kb)

Additional file 4: Figure S2. KEGG pathway classifications of genes encoded by the C. werkmanii BF-6 genome based on the KEGG database. Functional classifications were assigned to a total of 3453 genes, and the numbers with each classification are indicated. (DOCX $111 \mathrm{~kb}$ )

Additional file 5: Figure S3. Functional classification of genes encoded by $C$. werkmanii BF-6 genome based on the COG database. A total of 4234 genes with orthologs in the COG database were classified and the numbers with each classification are indicated. (DOCX $44 \mathrm{~kb}$ )

Additional file 6: Table S3. Primers used for amplification of biofilm genes in the qRT-PCR assay. (DOCX $13 \mathrm{~kb}$ )

\section{Abbreviations}

CLSM: Confocal Laser Scanning Microscopy; COG: Cluster of Orthologous Groups of Proteins; EPS: Extracellular polymeric substance; SD: Standard deviations; GO: Gene Ontology; KEGG: Kyoto Encyclopedia of Genes and Genomes; PDO: 1, 3-propanediol 


\section{Acknowledgements}

Not applicable.

\section{Funding}

This work was funded by the Natural Science Foundation of Guangdong Province (No. 2015A030313713), and National Natural Science Foundation of China (No. 31500036)

\section{Availability of data and materials}

The genome of C. werkmanii BF- 6 has been deposited in the NCBI database under the accession number CP019986 (chromosome) and CP019987 (plasmid). The data set of Fig. 1 supporting the results of this article is available in the TreeBase (http://treebase.org/treebase-web/home.html) repository, under the accession URL http://purl.org/phylo/treebase/phylows/study/TB2:S21590.

\section{Authors' contributions}

GZ and QSS designed the experiments, did the data analysis and wrote the manuscript. HP and YSW did the CLSM and RT-PCR. XMH and XBX helped the data analysis and contributed to manuscript writing and revision. All authors read and approved the final manuscript.

\section{Ethics approval and consent to participate}

Not applicable.

\section{Consent for publication}

Not applicable.

\section{Competing interests}

The authors declare that they have no competing interests.

\section{Publisher's Note}

Springer Nature remains neutral with regard to jurisdictional claims in published maps and institutional affiliations.

\section{Author details}

'Guangdong Institute of Microbiology, Guangzhou, Guangdong 510070, People's Republic of China. ${ }^{2}$ State Key Laboratory of Applied Microbiology Southern China, Guangzhou, Guangdong 510070, People's Republic of China. ${ }^{3}$ Guangdong Provincial Key Laboratory of Microbial Culture Collection and Application, Guangzhou, Guangdong 510070, People's Republic of China. ${ }^{4}$ Guangdong Open Laboratory of Applied Microbiology, Guangzhou, Guangdong 510070, People's Republic of China.

\section{Received: 22 June 2017 Accepted: 4 October 2017}

\section{Published online: 10 October 2017}

\section{References}

1. Werkman CH, Gillen GF. Bacteria producing trimethylene glycol. J Bacteriol. 1932;23:167-82

2. Lipsky BA, Hook EW, Smith AA, Plorde JJ. Citrobacter infections in humans: experience at the Seattle veterans administration medical center and a review of the literature. Rev Infect Dis. 1980;2:746-60.

3. Hodges G, Degener C, Barnes W. Clinical significance of Citrobacter isolates. Am J Clin Pathol. 1978;70:37-40.

4. Arens S, Verhaegen J, Verbist L. Differentiation and susceptibility of Citrobacter isolates from patients in a university hospital. Clin Microbiol Infect. 1997:3:53-7.

5. Katzenellenbogen E, Kocharova NA, Korzeniowska-Kowal A, Bogulska M, Rybka J, Gamian A, Kachala W, Shashkov AS, Knirel YA. Structure of the glycerol phosphate-containing O-specific polysaccharide and serological studies on the lipopolysaccharides of Citrobacter werkmanii PCM 1548 and PCM 1549 (serogroup 014). FEMS Immunol Med Microbiol. 2008;54:255-62.

6. Doran TI. The role of Citrobacter in clinical disease of children: review. Clin Infect Dis. 1999;28:384-94.

7. Badger JL, Stins MF, Kim KS. Citrobacter freundii invades and replicates in human brain microvascular endothelial cells. Infect Immun. 1999;67:4208-15.

8. Macaskie LE, Empson RM, Lin F, Tolley MR. Enzymatically-mediated uranium accumulation and uranium recovery using a Citrobacter sp. immobilised as a biofilm within a plug-flow reactor. J Chem Technol Biotechnol. 1995;63:1-16.

9. Jeong BC, Hawes C, Bonthrone KM, Macaskie LE. Localization of enzymically enhanced heavy metal accumulation by Citrobacter sp. and metal accumulation in vitro by liposomes containing entrapped enzyme. Microbiology. 1997:143:2497-507.

10. Finlay JA, Allan VJM, Conner A, Callow ME, Basnakova G, Macaskie LE. Phosphate release and heavy metal accumulation by biofilm-immobilized and chemically-coupled cells of a citrobacter sp. pre-grown in continuous culture. Biotechnol Bioeng. 1999;63:87-97.

11. Maervoet VE, Beauprez J, De Maeseneire SL, Soetaert WK, De Mey M. Citrobacter werkmanii, a new candidate for the production of 1,3propanediol: strain selection and carbon source optimization. Green Chem. 2012;14:2168-78.

12. Maervoet VE, De Maeseneire SL, Soetaert WK, De Mey M. Unraveling the dha cluster in Citrobacter werkmanii: comparative genomic analysis of bacterial 1,3-propanediol biosynthesis clusters. Bioprocess Biosyst Eng. 2014;37:711-8.

13. Maervoet VE, De Maeseneire SL, Avci FG, Beauprez J, Soetaert WK, De Mey M. High yield 1,3-propanediol production by rational engineering of the 3 hydroxypropionaldehyde bottleneck in Citrobacter werkmanii. Microb Cell Factories. 2016;15:23.

14. Costerton JW, Lewandowski Z, Caldwell DE, Korber DR, Lappin-Scott HM. Microbial biofilms. Annu Rev Microbiol. 1995;49:711-45.

15. Kikuchi T, Mizunoe Y, Takade A, Naito S, Yoshida S. Curli fibers are required for development of biofilm architecture in Escherichia coli K-12 and enhance bacterial adherence to human uroepithelial cells. Microbiol Immunol. 2005:49:875-84

16. Dueholm MS, Albertsen M, Otzen D, Nielsen PH. Curli functional amyloid systems are phylogenetically widespread and display large diversity in operon and protein structure. PLoS One. 2012;7:e51274.

17. Kim SM, Lee HW, Choi YW, Kim SH, Lee JC, Lee YC, Seol SY, Cho DT, Kim J. Involvement of curli fimbriae in the biofilm formation of Enterobacter cloacae. J Microbiol. 2012:50:175-8.

18. Rinaudi L, Fujishige NA, Hirsch AM, Banchio E, Zorreguieta A, Giordano W. Effects of nutritional and environmental conditions on Sinorhizobium melilot biofilm formation. Res Microbiol. 2006;157:867-75.

19. Speranza B, Corbo MR, Sinigaglia M. Effects of nutritional and environmenta conditions on Salmonella sp. biofilm formation. J Food Sci. 2011;76:M12-6.

20. Li LJ, Zhou G, Shi OS, Chen YC, Chen YB, Ouyang YS, Hu WF. Identification and biofilm formation characterization of Citrobacter werkmanii isolated from industrial spoilage. Microbiology China. 2014;41:2-7.

21. Zhou G, Li L, Shi Q, Ouyang Y, Chen Y, Hu W. Effects Of nutritional and environmental conditions on planktonic growth and biofilm formation for Citrobacter werkmanii BF-6. J Microbiol Biotechnol. 2013;23:1673-82.

22. Zhou G, Shi QS, Huang XM, Xie XB. Proteome responses of Citrobacter werkmanii BF-6 planktonic cells and biofilms to calcium chloride. J Proteome. 2016:133:134-43.

23. De Gregorio E, Del Franco M, Martinucci M, Roscetto E, Zarrilli R, Di Nocera PP. Biofilm-associated proteins: news from Acinetobacter. BMC Genomics. 2015;16:933

24. Hermans K, Roberfroid S, Thijs IM, Kint G, De Coster D, Marchal K, Vanderleyden J, De Keersmaecker SCJ, Steenackers HP. FabR regulates Salmonella biofilm formation via its direct target FabB. BMC Genomics. 2016;17:253

25. Brenner D, Grimont P, Steigerwalt A, Fanning G, Ageron E, Riddle C. Classification of Citrobacter farmeri sp. nov., Citrobacter youngae sp. nov., Citrobacter sedlakii sp. nov., and three unnamed Citrobacter genospecies. Int J Syst Bacteriol. 1993;43:645-58.

26. Brenner DJ, O'Hara CM, Grimont PA, Janda JM, Falsen E, Aldova E, Ageron E, Schindler J, Abbott SL, Steigerwalt AG. Biochemical identification of Citrobacter species defined by DNA hybridization and description of Citrobacter gillenii sp. nov. (formerly Citrobacter genomospecies 10) and Citrobacter murliniae sp. nov. (formerly Citrobacter genomospecies 11). J Clin Microbiol. 1999;37:2619-24.

27. Schauer DB, Zabel BA, Pedraza IF, O'Hara CM, Steigerwalt AG, Brenner DJ. Genetic and biochemical characterization of Citrobacter rodentium sp. nov. J Clin Microbiol. 1995:33:2064-8.

28. Jackson DW, Simecka JW, Romeo T. Catabolite repression of Escherichia coli biofilm formation. J Bacteriol. 2002:184:3406-10.

29. Görke B, Stülke J. Carbon catabolite repression in bacteria: many ways to make the most out of nutrients. Nat Rev Microbiol. 2008;6:613-24.

30. Stoodley P, Sauer K, Davies DG, Costerton JW. Biofilms as complex differentiated communities. Annu Rev Microbiol. 2002:56:187-209.

31. Whiteley M, Bangera MG, Bumgarner RE, Parsek MR, Teitzel GM, Lory S, Greenberg E. Gene expression in Pseudomonas aeruginosa biofilms. Nature. 2001:413:860-4. 
32. Schembri MA, Kjærgaard K, Klemm P. Global gene expression in Escherichia coli biofilms. Mol Microbiol. 2003;48:253-67.

33. Labbate M, Queck SY, Koh KS, Rice SA, Givskov M, Kjelleberg S. Quorum sensing-controlled biofilm development in Serratia liquefaciens MG1. J Bacteriol. 2004;186:692-8.

34. Weber MM, French CL, Barnes MB, Siegele DA, McLean RJA. Previously uncharacterized gene, yjfO (bsmA), influences Escherichia coli biofilm formation and stress response. Microbiology. 2010;156:139-47.

35. Domka J, Lee J, Wood TK. YliH (BssR) and YceP (BssS) regulate Escherichia coli K-12 biofilm formation by influencing cell signaling. Appl Environ Microbiol. 2006;72:2449-59.

36. Waters CM, Lu W, Rabinowitz JD, Bassler BL. Quorum sensing controls biofilm formation in Vibrio cholerae through modulation of cyclic di-GMP levels and repression of vpsT. J Bacteriol. 2008;190:2527-36.

37. Srivastava D, Waters CM. A tangled web: regulatory connections between quorum sensing and cyclic di-GMP. J Bacteriol. 2012;194:4485-93.

38. Bobrov AG, Kirillina O, Perry RD. The phosphodiesterase activity of the HmsP EAL domain is required for negative regulation of biofilm formation in Yersinia pestis. FEMS Microbiol Lett. 2005;247:123-30.

39. Kirillina O, Fetherston JD, Bobrov AG, Abney J, Perry RD. HmsP, a putative phosphodiesterase, and HmsT, a putative diguanylate cyclase, control Hmsdependent biofilm formation in Yersinia pestis. Mol Microbiol. 2004;54:75-88.

40. Kim Y, Wang X, Ma Q, Zhang XS, Wood TK. Toxin-antitoxin systems in Escherichia coli influence biofilm formation through YjgK (TabA) and fimbriae. J Bacteriol. 2009;191:1258-67.

41. Barnhart MM, Chapman MR. Curli biogenesis and function. Annu Rev Microbiol. 2006;60:131-47.

42. Wood TK. Insights on Escherichia coli biofilm formation and inhibition from whole-transcriptome profiling. Environ Microbiol. 2009;11:1-15.

43. Labrie J, Pelletier-Jacques G, Deslandes V, Ramjeet M, Auger E, Nash JH, Jacques $M$. Effects of growth conditions on biofilm formation by Actinobacillus pleuropneumoniae. Vet Res. 2010;41:3.

44. Chin CS, Alexander DH, Marks P, Klammer AA, Drake J, Heiner C, Clum A, Copeland A, Huddleston J, Eichler EE. Nonhybrid, finished microbial genome assemblies from long-read SMRT sequencing data. Nat Methods. 2013;10:563-9.

45. Li R, Li Y, Kristiansen K, Wang JSOAP. Short oligonucleotide alignment program. Bioinformatics. 2008:24:713-4.

46. Luo R, Liu B, Xie Y, Li Z, Huang W, Yuan J, He G, Chen Y, Pan Q, Liu Y, et al. SOAPdenovo2: an empirically improved memory-efficient short-read de novo assembler. Gigascience. 2012;1:18.

47. Krumsiek J, Arnold R, Rattei T. Gepard: a rapid and sensitive tool for creating dotplots on genome scale. Bioinformatics. 2007;23:1026-8.

48. Delcher AL, Bratke KA, Powers EC, Salzberg SL. Identifying bacterial genes and endosymbiont DNA with glimmer. Bioinformatics. 2007;23:673-9.

49. Lukashin AV, Borodovsky M. GeneMark.Hmm: new solutions for gene finding. Nucleic Acids Res. 1998;26:1107-15.

50. Zdobnov EM, Apweiler R. InterProScan-an integration platform for the signature-recognition methods in InterPro. Bioinformatics. 2001;17:847-8.

51. Qin J, Li R, Raes J, Arumugam M, Burgdorf KS, Manichanh C, Nielsen T, Pons $\mathrm{N}$, Levenez F, Yamada TA. Human gut microbial gene catalog established by metagenomic sequencing. Nature. 2010;464:59-65.

52. Xiao B, Sun YF, Lian B, Chen TM. Complete genome sequence and comparative genome analysis of the Paenibacillus mucilaginosus K02. Microb Pathog. 2016:93:194-203.

53. Shukla SK, Rao TS. Effect of calcium on Staphylococcus aureus biofilm architecture: a confocal laser scanning microscopic study. Colloid Surf BBiointerfaces. 2013;103:448-54

54. Heydorn A, Ersbøll BK, Hentzer M, Parsek MR, Givskov M, Molin S. Experimental reproducibility in flow-chamber biofilms. Microbiology. 2000; 146:2409-15.

55. Heydorn A, Nielsen AT, Hentzer M, Sternberg C, Givskov M, Ersbøll BK, Molin S. Quantification of biofilm structures by the novel computer program COMSTAT. Microbiology. 2000;146:2395-407.

56. Livak KJ, Schmittgen TD. Analysis of relative gene expression data using real-time quantitative PCR and the $2^{-\Delta \Delta C t}$ method. Methods. 2001;25:402-8.

57. Chan GF, Gan HM, Rashid NAA. Genome sequence of Citrobacter sp. strain A1, a dye-degrading bacterium. J Bacteriol. 2012;194:5485-6.

58. Kumar S, Kaur C, Kimura K, Takeo M, Raghava GPS, Mayilraj S. Draft genome sequence of the type species of the genus Citrobacter, Citrobacter freundii MTCC 1658. Genome Announc. 2013;1:e00120-12.
59. Lenz A, Tomkins J, Fabich AJ. Draft genome sequence of Citrobacter rodentium DBS100 (ATCC 51459), a primary model of Enterohemorrhagic Escherichia coli virulence. Genome Announc. 2015;3:e00415-5.

60. Basra P, Koziol A, Wong A, Carrillo CD. Complete genome sequences of Citrobacter braakii strains GTA-CB01 and GTA-CB04, isolated from ground beef. Genome Announc. 2015;3:e01307-14.

\section{Submit your next manuscript to BioMed Central and we will help you at every step:}

- We accept pre-submission inquiries

- Our selector tool helps you to find the most relevant journal

- We provide round the clock customer support

- Convenient online submission

- Thorough peer review

- Inclusion in PubMed and all major indexing services

- Maximum visibility for your research

Submit your manuscript at www.biomedcentral.com/submit

) Biomed Central 